Ingeniare. Revista chilena de ingeniería, vol. $15 \mathrm{~N}^{\circ} 1,2007$, pp. 111-117

\title{
ONDAS SOLITÓNICAS EN INTERFACES ABIÓTICAS-BIÓTICAS BASADAS EN FORMULACIÓN DE DRUDE PARA MEDIOS QUIRALES
}

\section{SOLITONIC WAVES IN BIOTIC-ABIOTIC INTERFACES BASED IN DRUDE FORMULATION FOR CHIRAL MEDIA}

\author{
Rodrigo Cancino L. ${ }^{1} \quad$ Mario Zamorano L. ${ }^{2} \quad$ Héctor Torres-Silva $^{3}$ \\ Recibido 6 de noviembre de 2006, aceptado 14 de marzo de 2007 \\ Received: November 6, 2006 Accepted: March 14, 2007
}

\begin{abstract}
RESUMEN
En este artículo se presenta un modelo teórico y la simulación computacional correspondiente, que permite analizar los efectos de la propagación de ondas solitónicas en medios biológicos esencialmente quirales. La quiralidad se modela, en este caso, bajo la formulación de Drude, mostrándose los rangos de validez del modelo propuesto. Este modelo considera los efectos no lineales y dispersivos del medio, debido a una dependencia espectral de la señal de entrada y la aproximación de campo cuasi-monocromático, para escribir la ecuación no lineal de Schrödinger y resolverla numéricamente mediante el método espectral de Fourier. Los resultados de nuestras simulaciones muestran el efecto de la variación del factor quiral sobre la impedancia del medio y sobre los coeficientes de transmisión y reflexión. Finalmente se discute, brevemente, la generalización del modelo de Drude para el caso de metamateriales.
\end{abstract}

Palabras clave: Quiralidad, ecuación no lineal de Schrödinger, formulación de Drude, medio biológico, tejido cerebral.

\section{ABSTRACT}

In this paper a theory model and the corresponding simulations which permit to analyze the solitonic wave propagation in biological media, is presented. The chirality is modeled as Drude's formulation, showing the validity rank of the model. The model considers the nonlinear and dispersive effects due to the spectral dependency of the input signal and the cuasy-monocromatic approach, so as to write the Schrödinger non-linear equation and solving it numerically by means of the spectral Fourier method. The numerical results show the effect of chiral factor variation on the media impedance, transmission and reflection coefficients. Finally, the generalization of the Drude's formulation for the metamaterial case, is briefly discussed.

Keywords: Chirality, non-linear Schrödinger equation, Drude's formulation, biological media, brain tissue.

\section{INTRODUCCIÓN}

La propagación de ondas en medios biológicos ha sido estudiada en profundidad en los últimos años, debido al resurgimiento de las tecnologías inalámbricas. Primeramente fueron los estudios a frecuencias correspondientes a la telefonía celular (900-1800 MHz); hoy en día con los sistemas inalámbricos del tipo WLL,
WIFI y WIMAX, el rango se ha extendido y las frecuencias de utilización van desde 1 a 100 [GHz]. Uno de los sustentos más serios que abre esta línea de investigación es el hecho que, en la actualidad, estamos expuestos a recibir, durante cinco minutos, más radiación que la recibida a lo largo de toda la vida de nuestros antepasados. Los estudios que han dado lugar a las normas sobre las potencias que deben emitir los aparatos celulares,

\footnotetext{
1 Programa de Magíster en Telecomunicaciones. Universidad de Tarapacá. Arica, Chile.

2 Escuela de Ingeniería Eléctrica-Electrónica. Universidad de Tarapacá. Arica, Chile.

3 Instituto de Alta Investigación. Universidad de Tarapacá. Arica, Chile.mhzlucero@uta.cl
} 
$(\mathrm{SAR})^{4}$, se obtienen mediante mediciones realizadas en laboratorios con materiales homogéneos de igual permitividad eléctrica y permeabilidad magnética, los que representan los diversos medios biológicos [ $\left[\begin{array}{ll}1 & \text { y }\end{array}\right]$. Los medios biológicos presentan simetrías moleculares que producen efectos que han sido descritos mediante variadas formulaciones. Este artículo considera que el medio de propagación es altamente dispersivo, la potencia de entrada es elevada, de manera que los modos no lineales de la polarización son incluidos en la ecuación de propagación y que además la conformación molecular presenta un giro a la izquierda o a la derecha. Esta última particularidad del medio se presenta como quiralidad, debido a su estructura constituyente, propia de los medios ópticamente activos. Las formulaciones de quiralidad dan cuenta de la rotación del plano de polarización debido a la birrefringencia circular, presente en medios biológicos tales como proteínas, lípidos, ácido nucleico, aminoácidos, $\mathrm{ADN}$, vitaminas, hormonas y en general en la mayoría de las sustancias naturales. De particular importancia para nuestro trabajo son los tejidos del cerebro. Al respecto, se han realizado estudios a partir de simulaciones realizadas sobre imágenes de resonancia magnética (MRI) de la cabeza humana, mediante el método de las diferencias finitas en el dominio del tiempo (FDTD), método que resuelve las ecuaciones de Maxwell directamente para un medio altamente no homogéneo. Este modelo, basado en imágenes MRI y técnica FDTD, ha sido usado ampliamente, inclusive considerando la quiralidad de la estructura del tejido cerebral [3]. Lamentablemente el método es una "caja negra" que no permite especular respecto del comportamiento de la onda en las diferentes interfaces y de ahí la necesidad de escribir la ecuación de propagación de acuerdo a las condiciones de contorno y analizar las respuestas.

En artículos anteriores, desarrollados por el grupo de trabajo, se ha demostrado que la ecuación de propagación, para medios no lineales dispersivos y quirales, está dada por [4 y 5]:

$$
\begin{aligned}
& \frac{\partial \vec{\Psi}_{ \pm}}{\partial z^{*}}-j \frac{1}{2}(1 \pm k T) k " \frac{\partial \vec{\Psi}_{ \pm}{ }^{2}}{\partial \tau^{2}}= \\
& -\vec{\Psi}_{ \pm} \frac{1}{2 k}\left\{\alpha \omega+j \omega^{2} \beta\left|\vec{\Psi}_{ \pm}\right|^{2}\right\}
\end{aligned}
$$

donde el primer término de la izquierda da cuenta de las variaciones de amplitud de la señal de entrada con respecto a la posición y el subíndice \pm significa ondas polarizadas circularmente a la izquierda o a la derecha respectivamente; el segundo término representa los efectos dispersivos del medio, ponderados por el grado de quiralidad $(\mathrm{T})$ o torsión de la molécula, mientras que en el segundo miembro se encuentra la atenuación debido a la conductividad finita y el término no lineal debido a la inclusión de la susceptibilidad de tercer orden. La relación de dispersión obtenida entrega rangos en los que el vector de radiación es antiparalelo con el sentido de propagación de la energía, es decir, el medio es metamaterial [4]. La ecuación (2) representa la ecuación homogénea para la condición metamaterial y destaca su carácter cuadrático y la explícita dependencia con la quiralidad del medio, donde $k_{ \pm}$representa el autovalor a la izquierda o a la derecha respectivamente.

$$
k_{ \pm}^{2}\left(1-\mu_{0} \varepsilon T^{2} \omega^{2}\right) \pm \varepsilon \mu_{0} \omega^{2}-2 \mu_{0} \varepsilon T \omega^{2} k_{ \pm}=0
$$

La ecuación (1) es dispersiva no-lineal, por lo que permite caracterizar la propagación de solitones. Dado que no tiene solución analítica, se debe hacer uso de un algoritmo numérico y uno de los métodos más eficientes para resolver este tipo de ecuaciones es el método espectral de Fourier [4 y 5].

De acuerdo a la conjetura de Frolish [8], la energía en un material biológico puede ser transferida, sin disipación, si se forman en el interior de las células estructuras solitónicas apropiadas, como las mostradas en las figuras la y $1 b$.

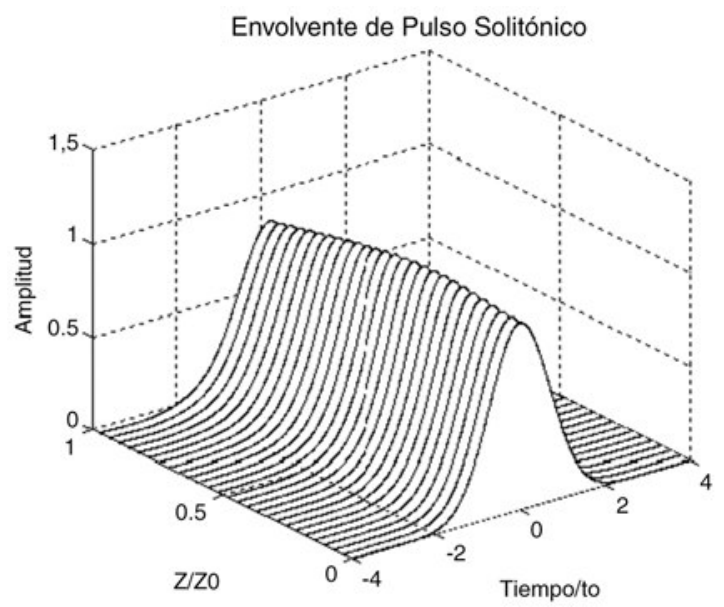

Figura 1a. Solitón en el dominio del tiempo propagándose en el espacio libre.

${ }^{4}$ Del inglés Specific Absorption Rate. SAR se usa como medida de la tasa de absorción de radiación de campo eléctrico en un área y masa determinada de tejido. 


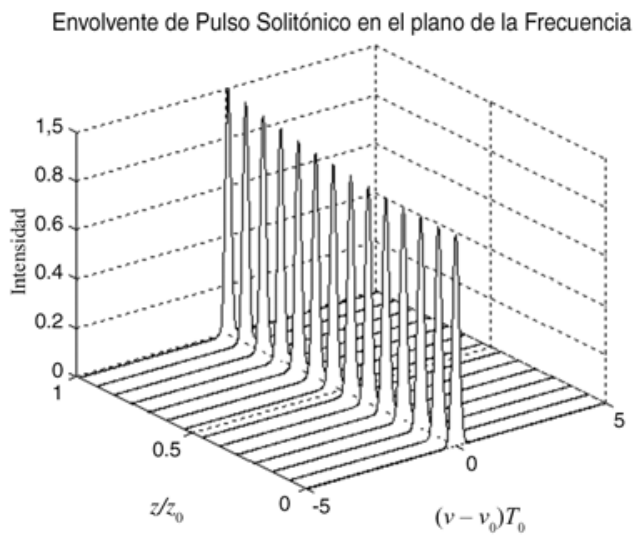

Figura 1b. Solitón en el dominio de la frecuencia propagándose en el espacio libre.

\section{INCIDENCIA DE ONDAS EN UNA INTERFAZ AIRE-TEJIDO ORGÁNICO}

Las relaciones que gobiernan el fenómeno de la interfaz se obtienen a partir de la formulación de Drude [10] para la quiralidad y están dadas por:

$$
\begin{gathered}
\vec{D}=\varepsilon(\vec{E}+T \nabla \times \vec{E}) \\
\vec{B}=\mu \vec{H} s
\end{gathered}
$$

Estas ecuaciones representan las relaciones constitutivas del medio y relacionan el desplazamiento eléctrico con la intensidad de campo eléctrico y la densidad de flujo magnético con la intensidad de campo magnético. Además, la ecuación (3) da cuenta de las rotaciones del plano de polarización eléctrico, de acuerdo al grado de torsión del medio dado por $T$. Para encontrar las expresiones bajo las condiciones de contorno estipuladas, se deben escribir las componentes de campo a ambos lados de la interfaz, para lo cual consideramos que la señal de entrada es cuasi-monocromática, y está dada por:

$$
\vec{E}_{ \pm}(z, t)=(\hat{i} \pm j \hat{j}) E_{0} e^{j\left(\omega t-k_{ \pm} z\right)}
$$

Ecuación que representa una onda circularmente polarizada a la izquierda o a la derecha, donde $k_{ \pm}=\frac{k}{1 \pm k T}$, es el autovalor de la ecuación (2). Como se requiere escribir las expresiones de las componentes de campo, se usa la ley de Faraday para hallar la expresión de la componente magnética y a partir de ella se encuentra la impedancia característica del medio:

$$
\frac{\vec{E}_{ \pm}}{\vec{H}_{ \pm}}=\eta_{q}= \pm \frac{j \omega \mu_{0}}{k_{ \pm}}= \pm j \eta_{0}(1 \pm k T)
$$

Ecuación que es concordante con los estudios realizados por H. Ammari [7], donde la impedancia del medio quiral lineal, bajo la formulación de Drude-Born-Fedorov, es imaginaria. En nuestro caso, además, la impedancia está ponderada por el parámetro quiral y las no linealidades están implícitas en la ecuación de propagación (ec.1).

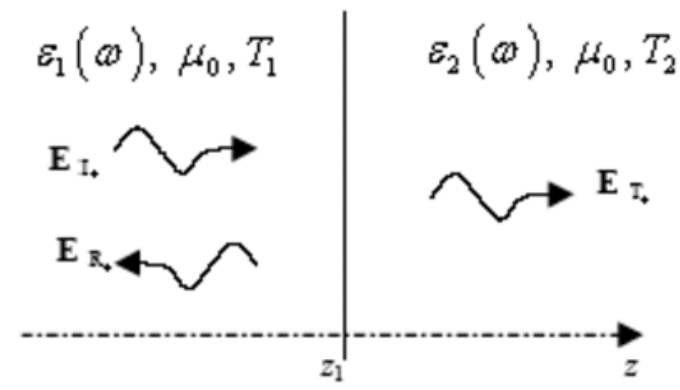

Figura 2. Problema de la interfaz.

Escribiendo las componentes de campo a ambos lados de la interfaz tenemos:

$$
\begin{aligned}
\vec{E}_{I \pm} & =\vec{\Psi}_{I \pm} e^{j\left(\omega t-k_{1 \pm} z\right)} \\
\vec{H}_{I \pm} & =\frac{\vec{\Psi}_{I \pm}}{\eta_{q 1}} e^{j\left(\omega t-k_{1 \pm} z\right.} \\
\vec{E}_{R \pm} & \left.=\vec{\Psi}_{R \pm} e^{j\left(\omega t+k_{1 \pm} z\right.}\right) \\
\vec{H}_{R \pm} & =-\frac{\vec{\Psi}_{R \pm}}{\eta_{q 1}} e^{j\left(\omega t+k_{1 \pm}\right)} \\
\vec{E}_{T \pm} & =\vec{\Psi}_{T \pm} e^{j\left(\omega t-k_{2 \pm} z\right)} \\
\vec{H}_{T \pm} & =\frac{\vec{\Psi}_{T \pm}}{\eta_{q 2}} e^{j\left(\omega t-k_{2 \pm} z\right)}
\end{aligned}
$$

y considerando las condiciones de contorno, es posible obtener:

$$
\begin{aligned}
& \vec{E}_{1}=\vec{\Psi}_{I \pm} e^{j\left(\omega t-k_{1+ \pm} z\right)}+\vec{\Psi}_{R \pm} e^{j\left(\omega t+k_{1 \pm} z\right)} ; \\
& \vec{E}_{2}=\vec{\Psi}_{T \pm} e^{j\left(\omega t-k_{2 \pm} z\right)}
\end{aligned}
$$




$$
\begin{aligned}
& \vec{H}_{1}=\frac{1}{\eta_{1}}\left(\vec{\Psi}_{I \pm} e^{j\left(\omega t-k_{1 \pm} z\right)}-\vec{\Psi}_{R \pm} e^{j\left(\omega t+k_{1 \pm} z\right)}\right) ; \\
& \vec{H}_{2}=\frac{1}{\eta_{2}} \vec{\Psi}_{T \pm} e^{j\left(\omega t-k_{2 \pm} z\right)}
\end{aligned}
$$

Finalmente, a partir de las ecuaciones anteriores, se obtiene:

$$
\begin{gathered}
\vec{\Psi}_{T \pm}=\frac{2 \eta_{2}}{\eta_{1}+\eta_{2}} \vec{\Psi}_{I \pm} e^{-j\left(k_{2 \pm}-k_{1 \pm}\right) z_{1}} \\
\vec{\Psi}_{R \pm}=\frac{\eta_{2}-\eta_{1}}{\eta_{2}+\eta_{1}} \vec{\Psi}_{I \pm} e^{-j 2 k_{1 \pm} z_{1}}
\end{gathered}
$$

De donde se deduce que los coeficientes de reflexión y de transmisión son:

$$
\begin{aligned}
& R_{ \pm}=\frac{\eta_{2}\left(1 \pm k T_{2}\right)-\eta_{1}\left(1 \pm k T_{1}\right)}{\eta_{2}\left(1 \pm k T_{2}\right)+\eta_{1}\left(1 \pm k T_{1}\right)} \\
& \tilde{T}_{ \pm}=\frac{2 \eta_{2}\left(1 \pm k T_{2}\right)}{\eta_{2}\left(1 \pm k T_{2}\right)+\eta_{1}\left(1 \pm k T_{1}\right)}
\end{aligned}
$$

Es clara la dependencia implícita de las expresiones obtenidas con el grado de torsión molecular. En forma gráfica se obtiene:

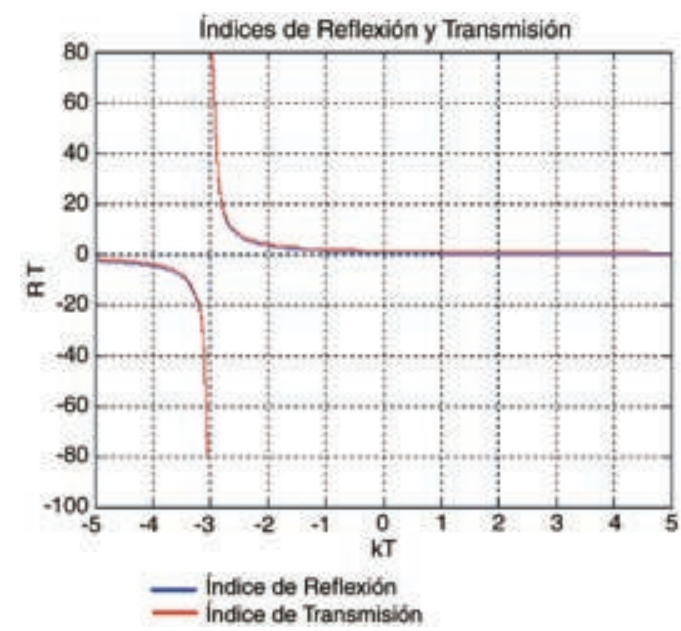

Figura 3. Respuesta del los índices de reflexión y transmisión para ondas RCP. $\eta_{1}=120 \pi ; \quad \eta_{1}=\eta_{2} ; \quad T_{2}=0 \quad T_{1} \neq 0$

A partir de la figura 3 se puede deducir un rango de validez, para el valor del producto $k T$, de modo que no se produzcan amplitudes físicamente inaceptables. La validez del modelo está también sustentada en el cumplimiento de la conservación de la energía, dada por $T-R=1$.

Para el caso de los valores mostrados en la figura 3 se presenta la siguiente gráfica del comportamiento de un solitón.
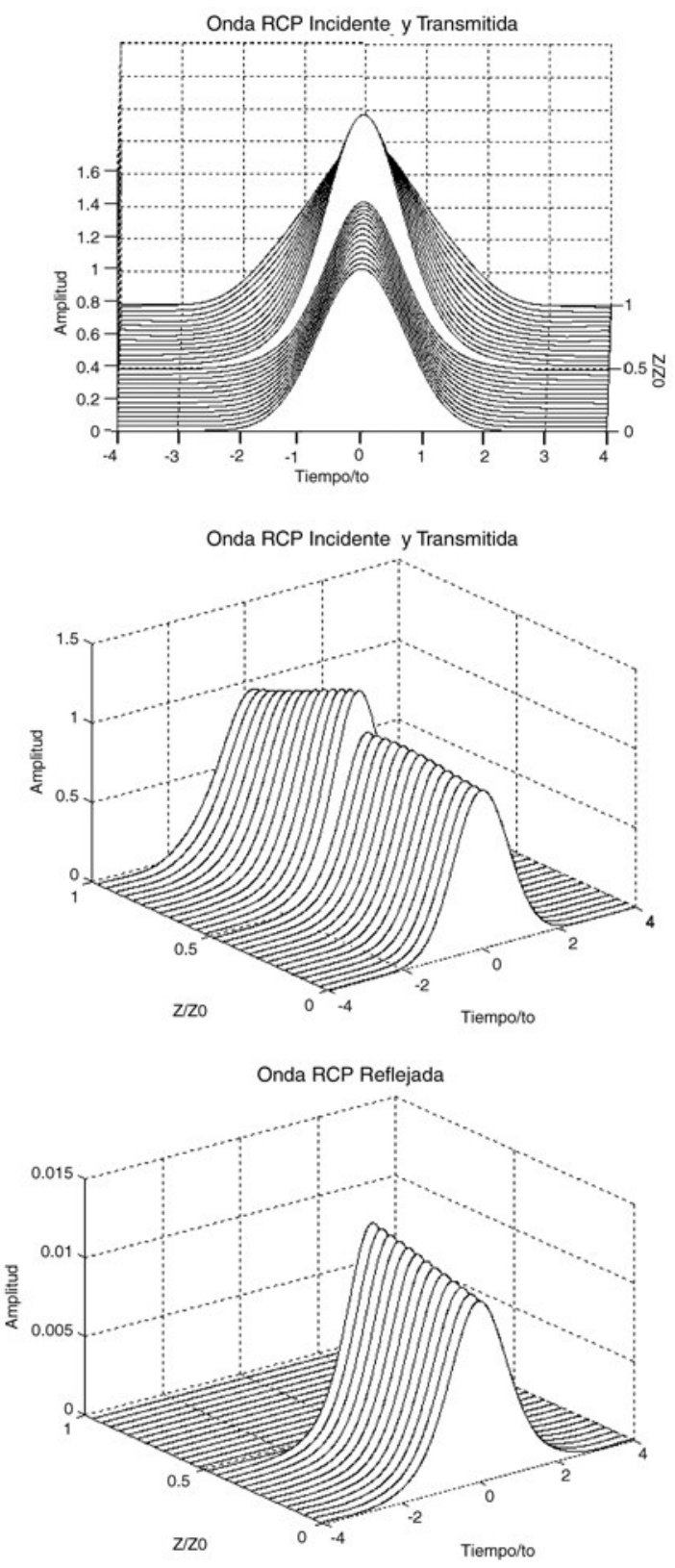

Figura 4. Propagación de ondas solitónicas en el plano del tiempo para $\eta_{1}=120 \pi ; \quad \eta_{2}=2 \eta_{1} ; \quad T_{2}=0 \quad T_{1} \neq 0$

En este caso se ve un incremento de la intensidad transmitida al medio 2 , la que se produce en desmedro de 
la componente magnética. El incremento del parámetro quiral del medio 2 producirá un aumento en la energía transmitida.

El tejido cerebral a $30[\mathrm{GHz}]$ presenta los siguientes valores para la conductividad y la permitividad:

$$
\sigma=27.5[\text { mho }], \varepsilon_{r}=18
$$

Las respuestas de las cantidades transmitidas se presentan en las figuras 5 y 6 .

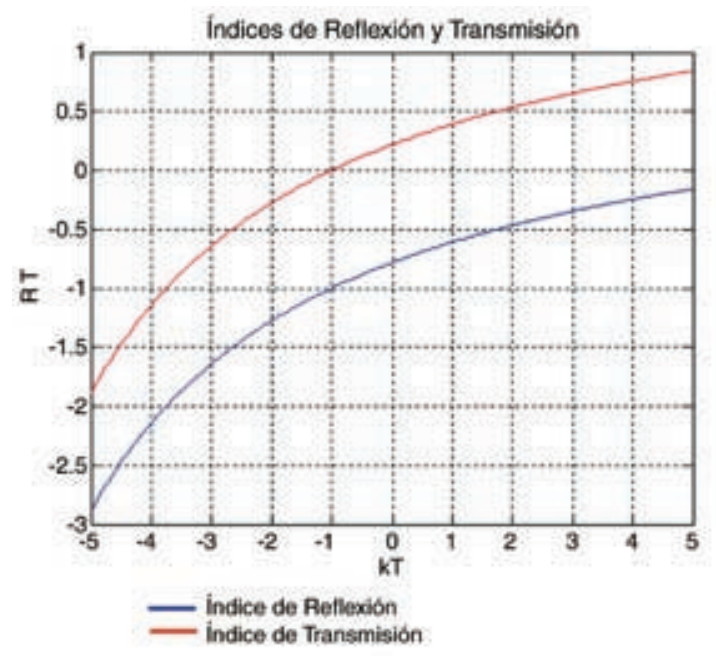

Figura 5. Comportamiento de los índices de reflexión y transmisión para los casos presentados con ondas RCP.

En la figura 5, para valores de $k T$ mayores que la unidad, se obtienen respuestas espectrales que denotan un incremento de la intensidad transmitida. La figura 6 muestra el comportamiento solitónico

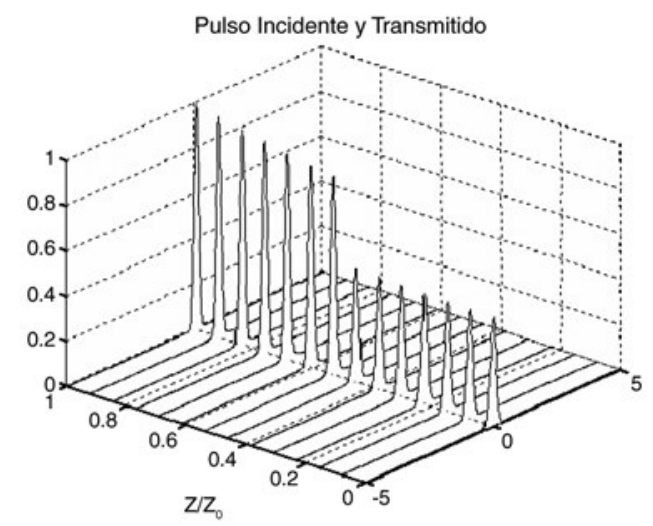

Figura 6. Respuesta frecuencial solitónica.

\section{COMPORTAMIENTO METAQUIRAL DE LOS MATERIALES}

En laboratorios de ciencia biomédica se ha descubierto una vía científica para producir un recubrimiento capaz de ocultar la materia, de tal modo que la luz y las microondas pasen aparentemente a través de ella, como si no hubiese nada interponiéndose. Una vez fabricado, este singular manto podría tener numerosos usos, como resulta fácil de prever, desde aplicaciones militares obvias, hasta otras menos previsibles en campos como las comunicaciones inalámbricas. Según los investigadores, del Imperial College de Londres y de la Escuela Pratt de Ingeniería en la Universidad de Duke, la capa podría esconder tan bien cualquier objeto que su presencia pasaría totalmente inadvertida para los observadores. En principio, la capa de invisibilidad podría ser fabricada con materiales artificiales compuestos, denominados metamateriales.

La capa actuaría como si se hubiera abierto un agujero en el espacio. Toda luz y otras ondas electromagnéticas, incidiendo en el área recubierta, serían guiadas por el metamaterial para emerger justo por el otro lado, como si hubieran atravesado un volumen vacío del espacio. Las ondas electromagnéticas rodearían el objeto escondido dentro del metamaterial y luego seguirían su curso normal, igual que hace el agua de un riachuelo al toparse con una piedra lisa.

Los metamateriales, demostrados inicialmente por Smith y sus colegas en el 2005 [11], pueden interactuar con la luz u otras ondas electromagnéticas de maneras muy precisas. Aunque el recubrimiento teórico ahora anunciado todavía tiene que ser creado, los investigadores de la Universidad de Duke están en camino de producir metamateriales con las propiedades adecuadas. Muchas son las aplicaciones de poder hacer invisible un cuerpo. Se podría permitir que los campos electromagnéticos pasasen "a través" de un objeto que los estuviera obstruyendo. Gracias a que eliminarían los efectos de las obstrucciones, tales recubrimientos también podrían mejorar las comunicaciones inalámbricas, que serían controladas por súper servidores de biosistemas operativos cuánticos y microcomputadores personales u ordenadores de mesa Empleando los mismos principios, una capa acústica podría servir como un escudo de protección, evitando la penetración de vibraciones, sonido e incluso ondas sísmicas. La metodología de diseño del grupo de expertos también puede encontrar numerosos usos diferentes del recubrimiento. Con el metamaterial apropiado, bien ajustado, la radiación electromagnética podría ser redirigida a voluntad para realizar virtualmente cualquier aplicación. Por ejemplo, en teoría podría llevar 
al desarrollo de metamateriales para enfocar la luz y proporcionar una lente más perfecta que cualesquiera que se haya fabricado antes. El desarrollo de los metamateriales abre un nuevo abanico de posibilidades, gracias a las propiedades electromagnéticas que proporcionan, las que son imposibles de encontrar en la naturaleza. El próximo gran desafío del equipo es una comprobación experimental de la invisibilidad a las ondas electromagnéticas en las frecuencias de las microondas. Para modelar tales medios es necesario generalizar el modelo de Drude en la forma:

$$
\vec{D}=\varepsilon(\vec{E}+T \nabla \times \vec{E}), \vec{B}=\mu(\vec{H}+T \nabla x \vec{H})
$$

$\mathrm{Y}$ en dominio de la frecuencia

$$
\vec{D}=\varepsilon(1 \pm k T) \vec{E}, \vec{B}=\mu(1 \pm k T) \vec{H}
$$

Para ondas circularmente polarizadas, Soukoulis y su equipo del Departamento de Energía del Laboratorio Ames de la Universidad Estatal de Iowa [12 y 13], están haciendo que la luz viaje hacia atrás a velocidades negativas (velocidad de fase de la onda que viaja en sentido contrario) que parecen más rápidas que la velocidad de la luz. De la ecuación de relación de dispersión se puede determinar qué modos pueden hacerlo.

Los materiales naturales refractan la luz o la radiación electromagnética, a la derecha del haz incidente, en diferentes ángulos y velocidades. Sin embargo, los metamateriales, también llamados materiales levógiros, permiten refractar la luz en un ángulo negativo, así que emerge en el lado izquierdo del haz incidente.

Esta característica de retraso curvo de los metamateriales permite aumentar la resolución en las lentes ópticas y podrían, potencialmente, conducir al desarrollo de superlentes planos, con la potencialidad de ver dentro de una célula humana y diagnosticar, por ejemplo, enfermedades de un bebé en su matriz.

Hasta la fecha, los metamateriales existentes funcionan en microondas o regiones infrarrojas lejanas del espectro electromagnético. Se ha fabricado por primera vez un metamaterial que tiene un índice de refracción negativo en 1.5 micrómetros [ 10 y 11]. Ésta es la longitud de onda más pequeña obtenida hasta ahora, son microscópicas y se pueden utilizar en las telecomunicaciones. También se ha demostrado que las dos velocidades de las longitudes de onda individuales, llamadas velocidad de fase y velocidad de grupo, son ambas negativas, lo que explica la capacidad de la luz de ser negativamente refractada, desafiando la teoría de Einstein de la relatividad y moviéndose aparentemente al revés y más rápidamente que la velocidad de la luz.

\section{CONCLUSIONES}

Se ha presentado el modelado y la simulación de la incidencia de microondas en una interfaz de material normal-metamaterial. Nuestros resultados numéricos demuestran que la formulación de Drude tiene un rango de validez, determinado por respuestas físicamente aceptables. La condición eminentemente imaginaria de la impedancia del medio quiral (molécula de ADN, proteínas, tejido celular) produce cambios de fase en las componentes de campo lo que se traduce en incrementos localizados de ondas transmitidas hacia el interior del tejido biótico, como consecuencia y dada la conductividad del mismo, se presentarían incrementos de temperatura debido a la disipación.

De manera específica se demostró que debido a la dependencia de la impedancia del medio con el factor quiral, los coeficientes de reflexión y transmisión también dependerán del factor quiral. Mediante simulaciones sistemáticas para $k T>0$ se demostró que la intensidad transmitida aumenta y para $k T<0$ el índice de transmisión disminuye.

Para el comportamiento de la onda en regímenes metaquirales, se producen una drástica disminución de la cantidad transmitida tal y como se desprende de las gráficas de la dependencia de las relaciones de contorno con el grado de torsión. Se sabe que para que existan reflexiones, los medios deben poseer diferentes parámetros dieléctricos-magnéticos, pero de acuerdo a lo formulado, basta una variación en el grado de torsión molecular para encontrar incrementos de amplitudes de campo.

Los trabajos siguientes se orientarán a la caracterización de interfaces en tejido biológico mediante el modelo propuesto, esto permitirá estudiar y evaluar los efectos de la radiación de microondas en medios biológicos y evaluar la interacción de las ondas con el tejido celular, especialmente en el caso del tejido cerebral.

Además, la generalización del modelo de Drude, permitirá modelar metamateriales que soporten ondas circularmente polarizadas con posibles aplicaciones en el área de las telecomunicaciones, militares y otras. 


\section{REFERENCIAS}

[1] A. Lakhtakia "Time Harmonic Electromagnetic Fields in Chiral Media". Springer-Verlag 15. 1989.

[2] C. Gabriel. "Dielectric properties of body tisúes at RF and Microwave frequencies".

http://www.brooks.af.mil/AFRL/HED/hedr/ reports/dielectric/Report/Report.html. 2005.

[3] F. Ortiz. "Análisis de la propagación de ondas electromagnéticas en medios quirales mediante el método FDTD". Tesis para optar al título de Ingeniero Civil Electrónico. Universidad de Tarapacá. 2002.

[4] M. Zamorano L. y H. Torres S. "Quirosolitones RCP y LCP en Óptica y Microondas en Régimen Normal y Metamaterial". Revista de la Facultad de Ingeniería - Universidad de Tarapacá. Vol. 12 $\mathrm{N}^{\circ}$ 1, pp. 49-57. 2004.

[5] H. Torres, M. Zamorano y C. Villarroel. "Efecto quiral en la ecuación de Schrödinger para una fibra óptica". Revista de la Facultad de Ingeniería - Universidad de Tarapacá. Vol. 73, pp. 35-40. 2004.

[6] M.P. Silverman. "Reflection and refraction at the surface of a chiral medium: Comparison of gyrotropic constitutive relations invarian or noninvariant under a duality transformation". Am. J. Phys. 54. 1969.
[7] H. Ammari. "Maxwell equations in periodic chiral structures". Math. Nachr. 251, pp. 3-18. 2003.

[8] H.F. Guttman, Bioelectrochemistry. Ed. F. Guttman and H. Keyzer. Plenum. New York. 1986.

[9] S. Tretyakov, I Nefedov, A. Sihvola, S. Maslovski, C. Simovski. "Waves and energy in chiral nihility". Journal of electromagnetic waves and applications. Vol. $17 \mathrm{~N}^{\circ}$ 5, pp. 695-706. 2003.

[10] V. Lindell, A.H. Sihvola, S.A. Tretyakov. "Electromagnetic in chiral and bi-isotropic media". Artech House. Second Edition. 2000.

[11] D.R. Smith, W.J. Padilla, D.C. Vier, S.C. NematNasser and S. Schultz. "Composite medium with simultaneously negative permeability and permittivity". Phy. Rev. Lett. Vol. $84 \mathrm{~N}^{\circ} 18$, pp. 4184-4187. May 2000.

[12] Gunnar Dolling, Christian Enkrich, Martin Wegener, Costas M. Soukoulis, Stefan Linden "Simultaneous Negative Phase and Group Velocity of Light in a Metamaterial". Science: Vol. 312 No 5775, pp. 892-894. 12 May 2006.

[13] Francis Elek and George V. Eleftheriades. "A two-dimensional uniplanar transmission-line metamaterial with a negative index of refraction". New J. Phys. Vol. 7, pp. 163. 2005. 